\title{
ON THE STATE CONSTRAINED MINIMAL TIME FUNCTION
}

\author{
R. J. STERN* \\ Department of Mathematics and Statistics \\ CONCORDIA UNIVERSITY \\ 1400 De Maisonneuve Blvd. \\ Montreal, Quebec H4B 1R6, Canada
}

April 8, 2003

\begin{abstract}
A standard class of finite dimensional control systems is considered, along with a state constraint set $S$ and a target set $\Sigma \subset S$. Under certain geometric assumptions on $S$ and a required $S$-constrained small time controllability property, a proximal Hamilton-Jacobi characterization of the $S$-constrained minimal time function to target $\Sigma$ is obtained.
\end{abstract}

\section{INTRODUCTION}

We shall consider a standard finite dimenisonal control system $\dot{x}(t)=f(x(t), u(t))$, $u(\cdot) \in U$, along with a state constraint $x(t) \in S$ and a target set $\Sigma \subset S$. Our goal is to provide, apparently for the first time, Hamilton-Jacobi (HJ) characterizations of the $S$-constrained minimal time function with target $\Sigma$. The unconstrained (that is, $S=\mathbb{R}^{n}$ ) form of this problem and related issues were settled in Wolenski and Zhuang [18]. There it was shown that under mild hypotheses, the unconstrained minimal time function is the unique function satisfying, among other properties, a proximal HJ equation on the complement of $\Sigma$, along with a boundary condition which takes the form of a certain proximal HJ inequality holding on $\Sigma$ itself. Other approaches to this issue, including viscosity methods, as well as further references, can be found in the books by Bardi and Capuzzo-Dolcetta [1] and Cannarsa and Sinestrari [2]. Of related interest are recent results of Clarke [4], where the proximal HJ equation was reconsidered, but in the absence of the aforementioned boundary condition. The solutions obtained to the proximal HJ equation in this new global framework were studied, and shown to be associated with geodesic trajectories.

As in [18] and [4], our methods here are based upon nonsmooth (proximal) monotonicityinvariance considerations as developed in Clarke, Ledyaev, Stern and Wolenski [5], [7]. But as will be seen, the imposition of a state constraint necessitates additional techniques. Of particular interest is the role played by a required $S$-constrained small time controllability hypothesis. Geometric sufficient conditions for the latter property will be provided as well, in the concluding comments.

State constrained control problems have received attention in recent years. In Clarke, Rifford and Stern [8], a state constrained Mayer problem was studied, and in Clarke and Stern [9], the problem of state constrained stabilization was considered. In both these

\footnotetext{
Key words: State constrained minimal time function, proximal Hamilton-Jacobi inequalities, small time controllability.

Mathematical Subject Classification: 49L99, 49N99, 49J52

* Research supported by the Natural Sciences Engineering Research Council of Canada and Le Fonds pour la Formation de Chercheurs et l'Aide à la Recherche du Québec.
} 
references, the emphasis was on the construction of feedback controls. In Clarke and Stern [10], the value function of the state constrained problem studied in [8] was characterized in proximal HJ terms; in this regard, see also Frankowska and Vinter [14] and Vinter [17]. As is the case in [10], the method here will rely upon state constrained trajectory tracking properties developed in [8], and the proximal characterizations can be reframed, as they were in [10], in the "constrained viscosity solution" framework introduced by Soner [16]. This will be remarked upon in the concluding comments.

We shall consider a control system of the form

$$
\dot{x}(t)=f(x(t), u(t)) \quad \text { a.e. }
$$

The state trajectory $x(\cdot)$ evolves in $\mathbb{R}^{n}$ and control functions $u(\cdot)$ are Lebesgue measurable functions $u: \mathbb{R} \rightarrow U$, where $U \subset \mathbb{R}^{m}$ is a compact control restraint set.

Hypotheses on the dynamics $f: \mathbb{R}^{n} \times U \rightarrow \mathbb{R}^{n}$ are as follows, and will be assumed to hold throughout. (The Euclidean norm is denoted by $\|\cdot\|$.)

(F1) The function $f$ is continuous and is locally Lipschitz in the state variable $x$, uniformly for $u \in U$; that is, for each bounded set $\Gamma \subset \mathbb{R}^{n}$, there exists $K_{\Gamma}>0$ such that

$$
\|f(x, u)-f(y, u)\| \leq K_{\Gamma}\|x-y\|
$$

whenever $(x, u)$ and $(y, u)$ are in $\Gamma \times U$.

(F2) The function $f$ satisfies a linear growth condition; that is, there exist positive numbers $c_{1}, c_{2}$ such that

$$
\|f(x, u)\| \leq c_{1}\|x\|+c_{2} \quad \forall(x, u) \in \mathbb{R}^{n} \times U .
$$

(F3) The velocity set $f(x, U)$ is convex for every $x \in \mathbb{R}^{n}$.

Under (F1)-(F2), for every initial state $\alpha$ and every control function $u(\cdot)$, there exists a unique trajectory $x(t)=x(t ; \alpha, u(\cdot))$ defined for all $t \geq 0$ and satisfying $x(0)=\alpha$. The imposition of (F3) is needed in order to have available the familiar property of sequential compactness of trajectories on compact time intervals, as is explained e.g. in [7]. See the concluding comments concerning the validity of our results when $f$ is only defined on $S \times U$, which is reasonable in $S$-constrained problems.

The next section provides a required result on $S$-constrained trajectory tracking. The main result is presented in $\S 3$, while $\S 4$ contains concluding comments. Prior to proceeding, we refer the reader to [7] for all the basic definitions and facts from nonsmooth analysis that will be required.

\section{S-CONSTRAINED TRAJECTORY TRACKING}

Geometric hypotheses on $S$ are now posited, and will be assumed to hold throughout.

(S1) $S$ is compact and wedged at each $x \in \operatorname{bdry}(S)$, meaning that at each boundary point $x$ one has pointedness of $N_{S}^{C}(x)$; that is, $N_{S}^{C}(x) \cap\left\{-N_{S}^{C}(x)\right\}=\{0\}$. Here $N_{S}^{C}(x)$ denotes the Clarke normal cone to $S$ at $x$. (This is equivalent to $T_{S}^{C}(x)$ having nonempty interior for each $x \in \operatorname{bdry}(S)$, where $T_{S}^{C}(x)$ denotes the Clarke tangent cone to $S$ at $x$.) 
(S2) The following "strict inwardness" condition holds:

$$
\min _{u \in U}\langle\zeta, f(x, u)\rangle<0 \quad \forall 0 \neq \zeta \in N_{S}^{C}(x), \quad \forall x \in \operatorname{bdry}(S) .
$$

The set $S$ being wedged at $x \in \operatorname{bdry}(S)$ is also referred to in the literature as epiLipschitzness of $S$ at $x$, since it is equivalent to $S$ being locally linearly homeomorphic to the epigraph of a Lipschitz function; see Rockafellar [15] and Clarke [3].

\section{Remark 2.1.}

(a) We will require the fact that when $S$ is wedged at each of its boundary points, then $S$ is the closure of its interior.

(b) (S1)-(S2) are sufficient but not necessary for $S$ to be weakly invariant; that is, for any initial state $\alpha \in S$, there exists a control $u(\cdot)$ producing a trajectory $x(t)=$ $x(t ; \alpha, u(\cdot))$ with $x(0)=\alpha$ and $x(t) \in S$ for all $t \geq 0$.

Given $r \geq 0$, an inner approximation of $S$ is given by

$$
S_{r}:=\left\{x \in S: d_{\hat{S}}(x) \geq r\right\} .
$$

Here $\hat{S}$ denotes the closure of the complement of $S$, and $d_{\Gamma}(\cdot)$ denotes the Euclidean distance function to a set $\Gamma$. (Note that $S_{0}=S$.) The geometry of inner approximations was studied in Clarke, Ledyaev and Stern [6], as well as in [8].

We will require the following $S$-constrained tracking result from the proofs of Proposition 3.13 and Theorem 3.10 in [8].

Lemma 2.2. There exists a constant $r_{0}>0$ satisfying the following two properties:

(a) Given $T>0$, there exists a constant $M=M(T)>0$ such that the following holds: Let $\alpha \in S, \alpha_{1} \in S$, and let $u_{0}(\cdot)$ be a control function on $[0, T]$ producing a trajectory which satisfies

$$
x\left(t ; \alpha_{0}, u_{0}(\cdot)\right) \in S \quad \forall t \in[0, T] .
$$

Then there exists a control function $u_{1}(\cdot)$ on $[0, T]$ which produces a trajectory which satisfies

$$
\left\|x\left(t ; \alpha_{1}, u_{1}(\cdot)\right)-x\left(t ; \alpha_{0}, u_{0}(\cdot)\right)\right\| \leq M\left\|\alpha_{1}-\alpha_{0}\right\| \quad \forall t \in[0, T]
$$

and

$$
x\left(t ; \alpha_{1}, u_{1}(\cdot)\right) \in S_{r} \quad \forall t \in[0, T] .
$$

(b) Given $T>0$, there exists $W=W(T)>0$ such that for any initial state $\alpha \in \operatorname{int}(S)$, if $r \in\left[0, r_{0}\right]$ is such that $r \leq d_{\hat{S}}(\alpha)$ and $u(\cdot)$ is a control function on $[0, T]$ such that

$$
x(t ; \alpha, u(\cdot)) \in S \quad \forall t \in[0, T],
$$

then there exists a control function $\bar{u}(\cdot)$ on $[0, T]$ such that

$$
\|x(t ; \alpha, \bar{u}(\cdot))-x(t ; \alpha, u(\cdot))\| \leq r W \quad \forall t \in[0, T],
$$

and

$$
x(t ; \alpha, \bar{u}(\cdot)) \in S_{r} \quad \forall t \in[0, T] .
$$




\section{The $S$-CONSTRAined Minimal TIME FUnCTION}

Let $\Sigma$ be a closed subset of $S$, and denote by $\Gamma_{S}$ the set of $\alpha \in S$ such that for some control $u(\cdot)$ and some $\hat{t} \geq 0$ one has

$$
x(\hat{t} ; \alpha, u(\cdot)) \in \Sigma
$$

and

$$
x(t ; \alpha, u(\cdot)) \in S \quad \forall t \in[0, \hat{t}] .
$$

In other words, $\Gamma_{S}$ consists of those initial states in $S$ which can be controlled in finite time to the target $\Sigma$ along an $S$-constrained trajectory. Denote by $\tau(\alpha)$ the infimal time from an initial state $\alpha \in \Gamma_{S}$ to the target $\Sigma$ along such a trajectory. By a standard sequential compactness of trajectories argument, the infimum is attained as a minimum. We go on to define an extended real valued function $T: \mathbb{R}^{n} \rightarrow[0,+\infty]$ as follows:

$$
T(\alpha):=\left\{\begin{array}{cc}
\tau(\alpha) & \text { if } \alpha \in \Gamma_{S} \\
+\infty & \text { otherwise }
\end{array}\right.
$$

$T(\cdot)$ is called the $S$-constrained minimal time function with respect to the target $\Sigma$. One can show that $T(x) \geq 0$ for all $x \in \mathbb{R}^{n}$ and $T(\alpha)=0$ if and only if $\alpha \in \Sigma$. Observe that there are two ways for $T(\alpha)$ to be non-finite:

(i) $\alpha \in S$, but $\Sigma$ is not reachable from $\alpha$ via an $S$-constrained trajectory; that is, $\alpha \in S \backslash \Gamma_{S}$.

(ii) $\alpha \notin S$.

Let us collect some basic properties of the functions $T(\cdot)$ and $g(\cdot, \cdot)$, where

$$
g(t, x):=t+T(x) .
$$

We denote the set of strictly positive real numbers by $\mathbb{R}_{+}$, and the complement of $\Sigma$ by $\Sigma^{c}$.

(LSC) $T(\cdot)$ is lower semicontinuous at every $\alpha \in \mathbb{R}^{n}$; that is

$$
T(\alpha) \leq \liminf _{\alpha^{\prime} \rightarrow \alpha} T\left(\alpha^{\prime}\right)
$$

(SI) $g(\cdot, \cdot)$ is strongly increasing on $\mathbb{R}_{+} \times \operatorname{int}(S)$. This means that for every $\alpha \in \operatorname{int}(S)$, for every trajectory of (1) with $x(0)=\alpha$, one has $g(t, x(t)) \geq T(\alpha)$ on $[0, s]$ for every $s \geq 0$ such that $x(t) \in \operatorname{int}(S)$ on $[0, s]$.

(WD) $g(\cdot, \cdot)$ is weakly decreasing on $\mathbb{R}_{+} \times \Sigma^{c}$. This means that for every $\alpha \in \Sigma^{c}$, there exists a trajectory of $(1)$ with $x(0)=\alpha$, for which $g(t, x(t)) \leq T(\alpha)$ on $[0, s]$ for every $s \geq 0$ such that $x(t) \in \Sigma^{c}$ on $[0, s]$.

Note that in (SI) and (WD), infinite values of $T(\cdot)$ and $g(\cdot, \cdot)$ are possible. In particular, in $(\mathrm{SI})$, note that if a trajectory in $\operatorname{int}(S)$ exits $\Gamma_{S}$, it cannot re-enter $\Gamma_{S}$ while remaining in $S$. Property (LSC) readily follows from a sequential compactness of trajectories argument. The strong increase property (SI) is a straightforward consequence of the 
Principle of Optimality, while the weak decrease property (WD) is due to the existence of $S$-constrained time-optimal trajectories from any startpoint $\alpha$ where $T(\alpha)$ is finite; that is, $\alpha \in \Gamma_{S}$. (Startpoints for which $T(\alpha)=+\infty$ trivially satisfy the weak decrease condition.)

We now introduce the lower Hamiltonian $h: \mathbb{R}^{n} \times \mathbb{R}^{n} \rightarrow \mathbb{R}$ by

$$
h(x, p):=\min _{u \in U}\langle p, f(x, u)\rangle
$$

where $\langle\cdot, \cdot\rangle$ denotes the standard inner product.

The next lemma provides proximal characterizations of (SI) and (WD) in terms of proximal HJ inequalities. It follows in a straightforward manner from Exercise 4.6.4(a) and Proposition 4.6.5 in [7].

\section{Lemma 3.1.}

(i) Property (SI) is equivalent to

$$
h\left(x, \partial_{P} T(x)\right)+1 \geq 0 \quad \forall x \in \operatorname{int}(S) .
$$

(ii) Property (WD) is equivalent to

$$
h\left(x, \partial_{P} T(x)\right)+1 \leq 0 \quad \forall x \in \Sigma^{c} .
$$

Here $\partial_{P}$ denotes the proximal subdifferential; recall that for an extended real-valued lower semicontinuous function $w: \mathbb{R}^{n} \rightarrow(-\infty,+\infty]$ and a point $x$ where $w(x)<+\infty$, $\zeta \in \partial_{P} w(x)$ if and only if there exists $\sigma=\sigma(x, \eta)$ such that

$$
w(y)-w(x)+\sigma\|y-x\|^{2} \geq\langle\zeta, y-x\rangle
$$

for all $y$ near $x$. Thus (10) says that

$$
h(x, \zeta)+1 \geq 0 \quad \forall \zeta \in \partial_{P} T(x), \quad \forall x \in \operatorname{int}(S),
$$

and (11) says that

$$
h(x, \zeta)+1 \leq 0 \quad \forall \zeta \in \partial_{P} T(x), \quad \forall x \in \Sigma^{c} .
$$

These inequalities hold vacuously when the proximal subdifferentials involved are empty, as is the case when $T(x)=+\infty$, but emptiness is not precluded even when $T(x)$ is finite.

Note also that $(10)$ and (11) together are equivalent to $T(\cdot)$ satisfying

$$
\begin{array}{cl}
h\left(x, \partial_{P} T(x)\right)+1=0 & \forall x \in\{\operatorname{int}(S)\} \backslash \Sigma, \\
h\left(x, \partial_{P} T(x)\right)+1 \geq 0 \quad \forall x \in\{\operatorname{int}(S)\} \cap \Sigma,
\end{array}
$$

and

$$
h\left(x, \partial_{P} T(x)\right)+1 \leq 0 \quad \forall x \in\{\operatorname{bdry}(S)\} \backslash \Sigma .
$$

We require the following definition, where the open unit ball is denoted $B:=\left\{z \in \mathbb{R}^{n}:\|z\|<1\right.$. $\}$ 
Definition 3.2. A function $\varphi: \mathbb{R}^{n} \rightarrow(-\infty,+\infty]$ is said to be $(\Sigma, S)$-continuous provided that there exist $\gamma_{\varphi}>0$ and a function $\omega_{\varphi}:\left[0, \gamma_{\varphi}\right] \rightarrow[0,+\infty)$ such that $\lim _{s \downarrow} \omega_{\varphi}(s)=0$ and $\varphi(x) \leq \omega_{\varphi}\left(d_{\Sigma}(x)\right)$ for all $x \in S \cap\left\{\Sigma+\gamma_{\varphi} B\right\}$.

In the absence of a state constraint (i.e. $S=\mathbb{R}^{n}$ ), the minimal time function being $\left(\Sigma, \mathbb{R}^{n}\right)$-continuous is often referred to as small time controllability. Accordingly, when the $S$-constrained minimal time function $T(\cdot)$ is $(\Sigma, S)$-continuous, then we say that $S$ constrained small time controllability holds.

Remark 3.3. Results concerning the small time controllability property in the absence of a state constraint may be found in [18], [1] and [2]. "Classical" trajectory tracking based on Gronwall's lemma features in these discusssions. Specifically, one has the following facts:

(a) Small time controllability is equivalent to $T(\cdot)$ being continuous on an open neighborhood of $\Sigma$. Specifically, on such a neighborhood one has

$$
|T(x)-T(y)| \leq \omega_{T}(\|x-y\|) .
$$

(b) When small time controllability holds, then $\Gamma$, the set of startpoints controllable to $\Sigma$, is open, and small time controllability is equivalent to the minimal time function $T(\cdot)$ being continuous on $\Gamma$.

In the state constrained case presently being considered, one can employ the tracking result of Lemma 2.2(a) in order to obtain the following analogs of (a) and (b):

(a') $S$-constrained small time controllability is equivalent to $T(\cdot)$ being continuous on $S \cap\{\Sigma+\gamma B\}$ for some $\gamma>0$. Specifically, on such a set, (15) holds.

(b') When $S$-constrained small time controllability holds, the set $\Gamma_{S}$ is open relative to $S$, and small time controllability is equivalent to the minimal time function $T(\cdot)$ being continuous on $\Gamma_{S}$.

The preceding remarks are not required in the following main result.

Theorem 3.4. Let (F1)-(F3), (S1)-(S2) hold, and assume that $S$-constrained small time controllability holds. Then there is a unique lower semicontinuous extended real valued function $\varphi(\cdot)$ which is $(\Sigma, S)$-continuous, bounded below on $\mathbb{R}^{n}$, identically 0 on $\Sigma$, identically $+\infty$ on $\Sigma^{c}$, and satisfies

$$
\begin{gathered}
h\left(x, \partial_{P} \varphi(x)\right)+1=0 \quad \forall x \in\{\operatorname{int}(S)\} \backslash \Sigma, \\
h\left(x, \partial_{P} \varphi(x)\right)+1 \geq 0 \quad \forall x \in\{\operatorname{int}(S)\} \cap \Sigma,
\end{gathered}
$$

and

$$
h\left(x, \partial_{P} \varphi(x)\right)+1 \leq 0 \quad \forall x \in \operatorname{bdry}(S) \backslash \Sigma .
$$

That function is the $S$-constrained minimal time function $T(\cdot)$. 
In order to understand the role played by $S$-constrained small time controllability and $(\Sigma, S)$-continuity in this result, suppose that $\Sigma \subset \operatorname{bdry}(S)$, and note that then the indicator function of $\Sigma$, namely

$$
\varphi(x)=\left\{\begin{array}{cc}
+\infty & \text { if } x \notin \Sigma \\
0 & \text { if } x \in \Sigma
\end{array}\right.
$$

will always satisfy the conditions set out in the theorem; in particular (16)-(18) hold vacuously. Therefore there is no hope of uniqueness holding in general, without further conditions.

Proof of the theorem: That $T(\cdot)$ satisfies the stated conditions has already been explained. Hence the uniqueness assertion remains to be verified. To this end, let $\varphi$ be as in the statement of the theorem. The proof will follow from the verification of two claims:

Claim 1: $T(\alpha) \leq \varphi(\alpha) \quad \forall \alpha \in S$.

To see this, note that (16) and (18) imply that the function $\tilde{g}(t, \alpha):=t+\varphi(\alpha)$ is weakly decreasing on $\mathbb{R}_{+} \times \Sigma^{c}$. We only need to consider $\alpha \in S \backslash \Sigma$, since if $\alpha \in \Sigma$ we have $T(\alpha)=\varphi(\alpha)=0$. Furthermore, we can assume that $\varphi(\alpha)<+\infty$, for otherwise the claim is trivially true.

Now, for $\alpha \in S \backslash \Sigma$ with $\varphi(\alpha)<+\infty$, weak decrease implies that for some trajectory of (1) with $x(0)=\alpha$, one has

$$
t+\varphi(x(t)) \leq \varphi(\alpha)
$$

as long as $x(t) \notin \Sigma$. Since $\varphi(\cdot)$ is globally bounded below and $\varphi(\alpha)$ is finite, we conclude that the trajectory eventually enters $\Sigma$. Then $T(\alpha)<+\infty$ and

$$
t+\varphi(x(t)) \leq \varphi(\alpha) \quad \forall t \in[0, T(\alpha)) .
$$

Upon invoking the lower semicontinuity of $\varphi(\cdot)$, we then have

$$
\begin{aligned}
T(\alpha) & =T(\alpha)+\varphi(x(T(\alpha))) \\
& \leq \liminf _{t \uparrow T(\alpha)}[t+\varphi((x(t)))] \\
& \leq \varphi(\alpha),
\end{aligned}
$$

proving the claim.

Claim 2: $T(\alpha) \geq \varphi(\alpha) \quad \forall \alpha \in S$.

Let us first consider the case where $\alpha \in \operatorname{int}(S)$. Without loss of generality, we can assume that $\alpha \in \Gamma_{S}$, for otherwise $T(\alpha)=+\infty$ and the claim will be trivial. Let $x(\cdot)$ be an $S$-constrained time-optimal trajectory of (1) to target $\Sigma$, with $x(0)=\alpha$. By the tracking result of Lemma 2.2(a), there exists $W=W(T(\alpha))>0$ such that for each sufficiently small $r>0$ there exists an $S_{r}$-constrained trajectory $x_{r}(\cdot)$ of $(1)$ on the interval $[0, T(\alpha)]$ with $x_{r}(0)=\alpha$ and

$$
\left\|x_{r}(T(\alpha))-x(T(\alpha))\right\| \leq r W .
$$


Then

$$
x_{r}(T(\alpha)) \in \Sigma+r W \bar{B},
$$

where $\bar{B}$ denotes the closure of the unit ball $B$.

In view of $(16)-(17)$, the function $\varphi(\cdot)$ is strongly increasing on $\operatorname{int}(S)$. Consequently

$$
T(\alpha)+\varphi\left(x_{r}(T(\alpha))\right) \geq \varphi(\alpha) .
$$

Since $\varphi(\cdot)$ is $(\Sigma, S)$-continuous, $(22)$ implies that the term $\varphi\left(x_{r}(T(\alpha))\right)$ can be made arbitrarily small by decreasing $r$, and we deduce that

$$
T(\alpha) \geq \varphi(\alpha) .
$$

It remains to consider the case where $\alpha \in \operatorname{bdry}(S)$. Again, we can assume that $T(\alpha)$ is finite, and we consider an $S$-constrained time-optimal trajectory $x(\cdot)$ of $(1)$ to target $\Sigma$, with $x(0)=\alpha$. In view of Remark 2.1(a), there exists a sequence $\left\{\alpha_{i}\right\}$ in the interior of $S$, such that $\alpha_{i} \rightarrow S$. From the preceding argument, we have that

$$
T\left(\alpha_{i}\right) \geq \varphi\left(\alpha_{i}\right)
$$

for each $i=1,2, \ldots$.

By the tracking result of Lemma 2.2(b), there exists $M=M(T(\alpha))>0$ such that for each $i$ there is a trajectory $x_{i}(\cdot)$ of $(1)$ for which $x_{i}(0)=\alpha_{i}$,

$$
\left\|x(t)-x_{i}(t)\right\| \leq M\left\|\alpha_{i}-\alpha\right\| \quad \forall t \in[0, T(\alpha)]
$$

and

$$
x_{i}(t) \in S \quad \forall t \in[0, T(\alpha)] .
$$

Since $x(T(\alpha)) \in \Sigma$, we have $x_{i}(T(\alpha)) \in \Sigma+M\left\|\alpha_{i}-\alpha\right\| \bar{B}$, and therefore the $S$-constrained small time controllability assumption, inequality (25), and the preceding case yield

$$
T(\alpha)+\omega_{T}\left(M\left\|\alpha_{i}-\alpha\right\|\right) \geq T\left(\alpha_{i}\right) \geq \varphi\left(\alpha_{i}\right)
$$

for each $i$. Since $\omega_{T}\left(M\left\|\alpha_{i}-\alpha\right\|\right) \rightarrow 0$ as $i \rightarrow+\infty$ and $\varphi(\cdot)$ is lower semicontinuous, we obtain $T(\alpha) \geq \varphi(\alpha)$, as required.

\section{Concluding COMments}

4.1. Sufficient conditions for $S$-constrained small time controllability. It is possible to replace the $S$-constrained small time controllability hypothesis in Theorem 3.4 by stronger geometric hypotheses. Specifically, we introduce the following condition, in which $\operatorname{proj}_{\Sigma}(x)$ denotes the set of closest points in $\Sigma$ to $x$, and $N_{S}^{P}(x)$ denotes the proximal normal cone to $S$ at $x$; see [7].

(S3) There exist $\delta_{1}>0, \delta_{2}>0$ such that the following holds: For each $x \in S \cap \Sigma^{c} \cap\left\{\Sigma+\delta_{1} B\right\}$, there exists $u(x) \in U$ for which

$$
\langle\eta, f(x, u(x))\rangle \leq 0 \quad \forall 0 \neq \eta \in N_{S}^{P}(x),
$$

and

$$
\langle x-y, f(x, u(x))\rangle \leq-\delta_{2}\|x-y\| \quad \forall 0 \neq y \in \operatorname{proj}_{\Sigma}(x)
$$


Intuitively, condition (S3) says that at each point $x$ in $S$ which is near but exterior to $\Sigma$, there exists a velocity $f(x, u(x))$ which "points into $S$ " (this is (29)) while simultaneously "pointing towards $\Sigma$ " (this is (30)). The "proximal aiming" technique of [5], [7] and Clarke and Wolenski [11] can now be brought to bear, whereby one shows that all limiting Euler solutions $x(\cdot)$ of the initial value problem

$$
\dot{x}(t)=f(x(t), u(x(t))), \quad x(0)=\alpha \in S \cap\left\{\Sigma+\delta_{1} B\right\},
$$

are bona fide trajectories of the control system (1) which remain in $S$ and, for some $\gamma_{1}>0, \gamma_{2}>0$, satisfy $T(\alpha) \leq \gamma_{1} d_{\Sigma}(\alpha)$.

The foregoing comments show that we can take $\omega_{T}(z)=\gamma z$, in the notation of Definition 3.2. Then by Remark 3.3(a'), $T(\cdot)$ is Lipschitz on $S \cap\left\{\Sigma+\gamma_{3} B\right\}$ for some $\gamma_{3}>0$. Similarly to Remark 3.3(b'), one can then invoke Lemma 2.2(b) in order to conclude that $T(\cdot)$ is Lipschitz on $\Gamma_{S}$.

A variant of Theorem 3.4 ensues.

Corollary 4.1. Let (F1)-(F3) and (S1)-(S3) hold. Then there is a unique lower semicontinuous extended real valued function $\varphi(\cdot)$ which is $(\Sigma, S)$-continuous, bounded below on $\mathbb{R}^{n}$, identically 0 on $\Sigma$, identically $+\infty$ on $\Sigma^{c}$, and satisfies

$$
\begin{array}{cl}
h\left(x, \partial_{P} \varphi(x)\right)+1=0 & \forall x \in\{\operatorname{int}(S)\} \backslash \Sigma, \\
h\left(x, \partial_{P} \varphi(x)\right)+1 \geq 0 & \forall x \in\{\operatorname{int}(S)\} \cap \Sigma,
\end{array}
$$

and

$$
h\left(x, \partial_{P} \varphi(x)\right)+1 \leq 0 \quad \forall x \in \operatorname{bdry}(S) \backslash \Sigma .
$$

That function is the $S$-constrained minimal time function $T(\cdot)$. Furthermore, $T(\cdot)$ is Lipschitz on $\Gamma_{S}$.

\subsection{Constrained viscosity formulation.}

Given a lower semicontinuous extended real valued function $w: \mathbb{R}^{n} \rightarrow(-\infty,+\infty$, we denote the Dini subderivate at $x$ in the direction $v$ by

$$
D w(x ; v):=\liminf _{\substack{t \downarrow 0 \\ u \rightarrow v}} \frac{w(x+t u)-w(x)}{t} .
$$

The D-subdifferential (or viscosity subdifferential) of $w(\cdot)$ at $x$ is the set

$$
\partial_{D} w(x):=\left\{\zeta \in \mathbb{R}^{n}:\langle\zeta, v\rangle \leq D w(x ; v) \forall v \in \mathbb{R}^{n}\right\} .
$$

Similarly to remarks made in [10], Theorem 3.4 and Corollary 4.1 hold true if $\partial_{P}$ is replaced by $\partial_{D}$ in the statement, because the $P$ - and $D$-subdifferentials approximate one another in a suitable sense. This in turn rests upon a theorem of Subbotin; see Proposition 3.4.5 in [7]. By Proposition 3.4.12 in [7], the $\partial_{D}$-form of the theorem can in turn be put into "constrained viscosity" terms, in Soner's terminology [16]; see also [1]. Specifically, (16) can be replaced by the condition

$$
h\left(x, g^{\prime}(x)\right)+1=0 \quad \forall x \in\{\operatorname{int}(S)\} \backslash \Sigma,
$$

for any $g \in C^{1}\left(\mathbb{R}^{n}\right)$ such that $\varphi-g$ has a local minimum at $x$. Furthermore, conditions (17) and (18) have analogous constrained viscosity reformulations. 
4.3. $S$-Restricted dynamics. Suppose that the dynamics are specified only for $x \in$ $S$; that is, $f: S \times U \rightarrow \mathbb{R}^{n}$. Such a restricted domain can be expected in control problems where the state in $S$-constrained. Again, similarly to [10], it can be shown that Theorem 3.4 remains true if (F1)-(F3) are replaced by the following two conditions:

(G1) The function $f$ is continuous on $S \times U$ and is Lipschitz in the state variable $x$, uniformly for $u \in U$; that is, there exists $K$ such that

$$
\|f(x, u)-f(y, u)\| \leq K\|x-y\|,
$$

whenever $(x, u)$ and $(y, u)$ are in $S \times U$.

(G2) The velocity set $f(x, U)$ is convex for every $x \in S$.

We can extend $f$ from $S \times U$ to $\mathbb{R}^{n} \times U$ as follows: Let $f_{i}$ denote the $i^{\text {th }}$ component function of $f, i=1,2, \ldots, n$. For each fixed $u \in U$, define a function $x \rightarrow \hat{f}_{i}(x, u)$ on $\mathbb{R}^{n}$ as follows.

$$
\hat{f}_{i}(x, u)=\min _{y \in S}\left\{f_{i}(y, u)+K\|y-x\|\right\} .
$$

Then $x \rightarrow \hat{f}_{i}(x, u)$ agrees with $f_{i}(x, u)$ on $S$, and is globally Lipschitz of rank $K$. We then extend $f$ componentwise by setting $f_{i}(x, u)=\hat{f}_{i}(x, u)$ for every $(x, u) \in \mathbb{R}^{n} \times U$. The resulting function $f: \mathbb{R}^{n} \times U \rightarrow \mathbb{R}^{n}$ is continuous on $\mathbb{R}^{n} \times U$ and is globally Lipschitz of rank $K$ in the state variable $x$, uniformly for $u \in U$; that is,

$$
\|f(x, u)-f(y, u)\| \leq K\|x-y\|,
$$

whenever $(x, u)$ and $(y, u)$ are in $\mathbb{R}^{n} \times U$. The global Lipschitz condition on the extended dynamics implies the linear growth condition needed for the global extendability of solutions, and possible nonconvexity of the velocity sets $f(x, U)$ for $x \notin S$ does not cause a problem.

\section{REFERENCES}

[1] M. Bardi and I. Capuzzo-Dolcetta. Optimal Control and Viscosity Solutions of Hamilton-Jacobi-Bellman Equations. Birkauser, Boston, 1997.

[2] P. Cannarsa and C. Sinestrari. Semiconcave Functions, Hamilton-Jacobi Equations, and Optimal Control, to appear.

[3] F. H. Clarke. Optimization and Nonsmooth Analysis. Wiley-Interscience, New York, 1983. Republished as Vol. 5 of Classics in Applied Mathematics, S.I.A.M., Philadelphia, 1990.

[4] F. H. Clarke. The Hamilton-Jacobi equation of minimal time control. Preprint, September, 2002.

[5] F. H. Clarke, Yu. S. Ledyaev, R. J. Stern, and P. R. Wolenski. Qualitative properties of trajectories of control systems: a survey. J. Dyn. Control Sys., 1:1-48, 1995.

[6] F.H. Clarke, Yu. S. Ledyaev, and R. J. Stern. Complements, approximations, smoothings and invariance properties. J. Convex Anal., 4:189-219, 1997. 
[7] F.H. Clarke, Yu. S. Ledyaev, R.J. Stern, and P.R. Wolenski. Nonsmooth Analysis and Control Theory, volume 178. Springer-Verlag, Graduate Texts in Mathematics, New York, 1998.

[8] F. H. Clarke, L. Rifford and R. J. Stern. Feedback in state constrained optimal control. ESAIM:COCV, 7:97-133, 2002.

[9] F. H. Clarke and R. J. Stern. State constrained feedback stabilization. S.I.A.M. J. Control Optim., to appear.

[10] F. H. Clarke and R. J. Stern. Hamilton-Jacobi characterization of the state constrained value. Nonlinear Analysis (TMA), to appear.

[11] F. H. Clarke, P. R. Wolenski. Control of systems to sets and their interiors. J. Optim. Th. Appl., 8:3-23, 1996.

[12] F. Forcellini and F. Rampazzo. On nonconvex differential inclusions whose state is constrained in the closure of an open set. Applications to dynamic programming. Differential and Integral Equations, 12:471-497, 1999.

[13] H. Frankowska and F. Rampazzo. Filippov's and Filippov-Wazewski's theorems on closed domains. J. Diff. Eq., 161:449-478, 2000.

[14] H. Frankowska and R. B. Vinter. Existence of neighboring feasible trajectories: Applications to dynamic programming for state-constrained optimal control problems. J. Optim. Th. Appl., 104:21-40, 2000.

[15] R. T. Rockafellar. Clarke's tangent cones and boundaries of closed sets in $\mathbb{R}^{n}$. Nonlinear Anal., 3:145-154, 1979.

[16] M. Soner. Optimal control problems with state-space constraints I. S.I.A.M. J. Contr. Optim., 24:551-561, 1986.

[17] R. B. Vinter. Optimal Control. Birkauser, Boston, 2000.

[18] P. R. Wolenski and Y. Zhuang. Proximal analysis and the minimal time function. S.I.A.M. J. Contr. Optim., 36:1048-1072, 1998 